\title{
Interactive comment on "Precipitable water vapor content from ESR/SKYNET Sun-sky radiometers: validation against GNSS/GPS and AERONET over three different sites in Europe" by Monica Campanelli et al.
}

Monica Campanelli et al.

m.campanelli@isac.cnr.it

Received and published: 7 November 2017

See the attached pdf file

Please also note the supplement to this comment:

https://www.atmos-meas-tech-discuss.net/amt-2017-221/amt-2017-221-AC2- 